\title{
UNBO: Sistema de asistencia contextual al adulto mayor
}

\author{
Julio Rafael Bagur, Sofía Bonifasi Asturias, Rodrigo \\ José Cano
}

Publicado: 21 Septiembre 2016

\begin{abstract}
Resumen
Una de las grandes tendencias demográficas de la sociedad moderna es el hecho que estamos envejeciendo, se espera que para el 2050 crezca en 5\% el número de adultos mayores llegando a ser 1/5 de la población total del planeta [6]. Esto conlleva gran cantidad de retos a enfrentar ya que envejecer trae consigo nuevos problemas difíciles de prevenir en cualquier entorno social o comercial. Esto fue nuestra inspiración para desarrollar una plataforma multicontextual para asistir a todo tipo de adulto mayor sin importar sus impedimentos físicos. Llamamos a este sistema UNBO, por el hecho que toma la forma de un solo botón que permite pedir asistencia en cualquier ambiente donde se encuentre el usuario; en dos modos: ayuda común es decir, asistencia para movilizarse, específicos al contexto como llevar bienes $\mathrm{u}$ orientarse, $\mathrm{y}$ un modo de emergencia para asistencias de salud con personal capacitado. Una solución sencilla, elegante y clara a una multitud de problemas; todo con un solo botón.
\end{abstract}

Palabras clave: Facilidad; Interacción con el Ambiente; Entrevista Contextual; Bajo Costo; Adulto Mayor; Adaptabilidad; Asistencia.

\section{Introducción}

La llegada a la tercera edad conlleva una gran cantidad de consecuencias, de variante complejidad y no por ello dejan de existir responsabilidades y deberes que realizar. Todas estas interacciones fuera de entornos de confianza generalmente no toman en consideración este sector de la población de cualquier nación, especialmente en países con altos índices de pobreza como Guatemala y México donde arriba del $80 \%$ de la población tiene menos de 64 años [1].

Facilitar el día a día de estas personas queda relegado como una problemática de menor importancia frente a problemas más presentes y críticos como la desnutrición crónica y la falta de educación a gran escala. En un país donde estos problemas llevan décadas sin resolverse no se puede esperar que se logre resolver a problemáticas más avanzadas que incluyen el cuidado y protección del adulto mayor.

Gran mayoría de comercios y áreas sociales dentro de la ciudad poseen poca cantidad de facilidades para personas

\footnotetext{
Bagur, JR., Bonifasi Asturias, S., Cano, RJ.

Computer Science

Universidad Francisco Marroquín

Guatemala,Guatemala

Email: jbagur@ufm.edu, sofiabonifasi@ufm.edu, rjcano@ufm.edu
}

discapacitadas o mayores, más allá de parqueos para discapacitados o motonetas para personas de movilidad reducida. Esto lo observamos de primera mano al realizar una fase de investigación previa a la definición del proyecto a completar. Entrevistamos a gran cantidad de adultos mayores en contextos de vida distintos; familiares con cuidado en casa, viviendo solos o en pareja, adultos en hogares con facultades distintas; realizando preguntas sobre su relación con la tecnología moderna, dificultades a las que se enfrentan en su vida diaria, sus expectativas en la sociedad moderna y concretamente problemas que creyeran se puede solucionar con tecnología.
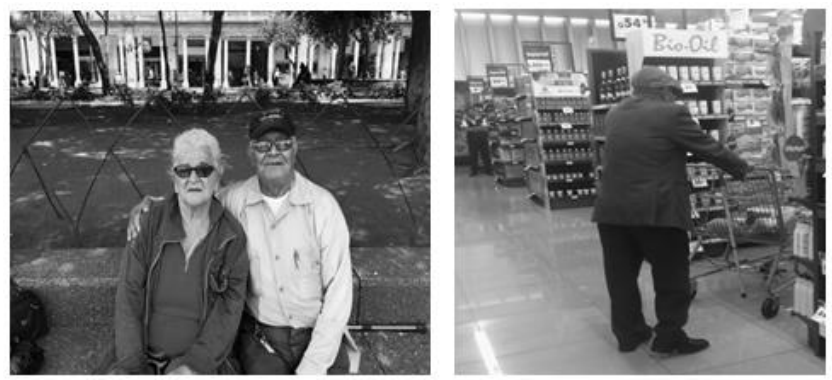

Figura 1. Personas Entrevistadas durante Fase de Investigación

Una respuesta común que nuestros entrevistados expresaron fue un profundo desinterés por aprender a utilizar nueva tecnología. Esto debido a que dichas personas mencionaron que generalmente solo buscan comunicarse vía teléfono con su grupo de amigos, conocidos o familiares, cualquier otro tipo de relación interpersonal a través de redes sociales o servicios de llamada en línea como Skype no les llama la atención en lo más mínimo.

Derivado de nuestras entrevistas y observaciones obtuvimos bastante información concreta y personal que nos permite visualizar una solución que facilite el uso de ambientes comerciales y sociales, creando una plataforma para auxiliar al adulto mayor en cualquier contexto, conectando con una persona capacitada y sin crear complicación innecesaria.

Nuestra propuesta combina simplicidad y accesibilidad en cualquier contexto y toma la forma de un solo botón: UNBO.

\section{Descripción del problema}

Durante la fase de observación se visitaron ambientes comerciales (supermercados, tiendas de conveniencia, tiendas por mayoreo, centros comerciales) de clase alta, media y baja en la Ciudad de Guatemala. Se observó dificultad en personas mayores para 
encontrar, transportar y comprar en estos ambientes por falta de recursos para asistirle.

Se observó que las personas mayores prefieren dirigirse a una persona a la hora de pedir ayuda en búsqueda de precios y movilización de objetos que estén fuera de su alcance por altura o peso, esto consumiendo gran cantidad de tiempo y poniendo en riesgo a adultos de movilidad reducida, ya que no se cuenta con la infraestructura adecuada para ayudar a personas que lleven un bastón, andador o una silla de ruedas.

Guatemala cuenta con un alto porcentaje de personas con discapacidad, del $4.6 \%$ que compone las personas mayores de 70 años en la población de Guatemala, el.008\% son discapacitadas [2] de acuerdo a la Encuesta Nacional de Discapacidad de 2005. Esto se traduce a más de 180,000 personas discapacitadas la mayoría siendo del casco urbano, todas estas personas se deben tomar en cuenta para crear una solución que efectivamente cubra a la gran mayoría de ciudadanos guatemaltecos de edad avanzada y adicionalmente incluya a personas discapacitadas fuera de este rango de edad.
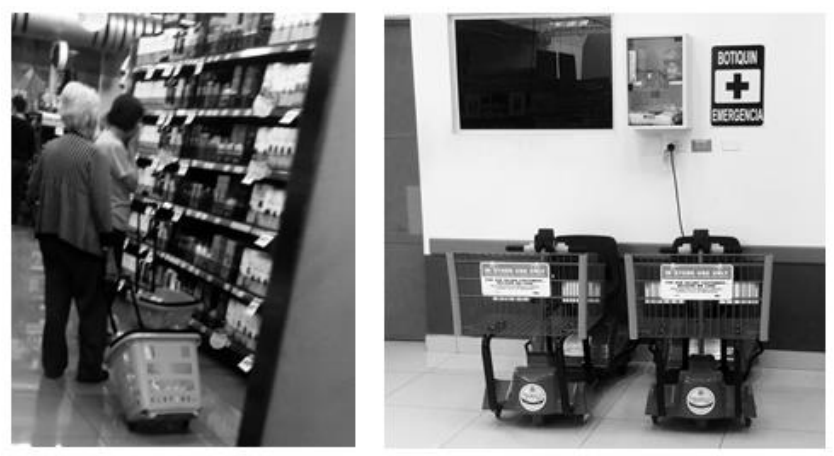

Figura 2. Facilidades presentes en comercios

\section{Desarrollo de UNBO}

Para llevar a cabo el desarrollo de UNBO, se tomaron en cuenta las guías de metodología del Diseño Centrado en el Usuario, utilizando como orientación el ISO 9241-11 [3] de Usabilidad para determinar el set de cualidades que creemos adecuadas para nuestro producto y se mantenga un estándar de excelencia en el mismo.

El estándar comprende de 6 principios que se requieren en el Diseño Centrado en el Usuario efectivo:

- Entendimiento del Usuario y Su Contexto

- Involucrar al Usuario a Través del Proceso

- Guiado por Evaluación del Usuario

- Iterativo

- Comprende la Experiencia Entera

- Multidisciplinario

Esto fue una guía para seleccionar los procesos a llevar a cabo para facilitar el proceso de desarrollo iterativo para desarrollar efectivamente nuestra solución al problema de la falta de facilidades para personas mayores en la sociedad guatemalteca.

\subsection{Entendimiento del usuario y su contexto}

Para comprender las necesidades y carencias del adulto mayor, se realizaron alrededor de 25 entrevistas contextuales a personas dentro de los ambientes de comercio, instituciones de cuidado especializado en el adulto mayor, áreas públicas de la ciudad de Guatemala y suburbios aledaños en manera de estudios de campo donde luego de conversar con los transeúntes y residentes de estas áreas se llegaron a las siguientes conclusiones:
- Falta de facilidades para personas mayores (anaqueles altos, falta de asistencia, material de lectura inadecuado, personas poco capacitadas para tratar con necesidades especiales, etc.)

- Falta de facilidades para personas discapacitadas. (Gradas sin opción a rampa, necesidad de maniobrar con dos manos, espacios reducidos que no permiten girar sillas de ruedas, superficies resbalosas.)

- Falta de motivación intrínseca para aprender tecnología "innecesaria"

- Deseo de interactuar con personas al momento de requerir asistencia.

A partir de estas conclusiones se crearon Personas [4] que pudiéramos tomar en cuenta para comenzar propiamente el diseño de los prototipos a evaluar en el proceso iterativo:

Tabla 1. Descripción de Personas para análisis UCD

\begin{tabular}{|l|l|}
\hline Persona 1 & Persona 2 \\
\hline Clarisa Gutierrez & Miguel Gonzales \\
\hline 65 años & 70 años \\
\hline Silla de ruedas & Movilidad ideal \\
\hline Clase baja & Clase media-alta \\
\hline Vendedora, pensionada & Retirado \\
\hline
\end{tabular}

\subsection{Involucrar al usuario a través del proceso}

Luego de determinar el modelo de funcionalidad de nuestra solución se regresó a una segunda fase de entrevistas con la meta de encontrar funciones no previstas y servir de guía en la determinación de la apariencia física del producto, problemas de ergonomía en productos que ya utilizan y que se pueda mejorar en UNBO, brindándonos con los siguientes hallazgos que nos guiaron a diseñar los primeros prototipos físicos:

- No creen conveniente que se tenga más de un botón porque se puede volver confuso en una situación de emergencia.

- Prefieren que el producto sea portátil y ligero; que se pueda mantener al alcance fácilmente, sin embargo grupos de edades avanzadas no deseaban que fuera algo semi-permanente en el cuerpo como una pulsera o un pendiente en una cadena.

- Tienen preferencia a que tenga un tamaño cómodo al agarrar en una mano, fabricado en un material resistente y de textura rugosa que facilite el agarre y que tenga colores brillantes en el botón para que contraste.

- Los colaboradores que tomen el rol de asistencia en los lugares donde UNBO va a actuar deben tener gran paciencia con el usuario y estar capacitados para asistir a adultos mayores con capacidades distintas de manera efectiva.

\subsection{Iterativo}

Basados en los hallazgos determinados por la segunda fase de entrevistas, llegamos a la conclusión que un botón con dos funciones: un pulso sencillo es llamada y un pulso sostenido es emergencia, es lo más adecuado y concuerda con lo que se conocía hasta el momento sobre el usuario. Se comenzó a diseñar prototipos de baja fidelidad comenzando con dibujos señalando las funciones y llevándolo a adultos mayores a que nuevamente lo evaluaran. 


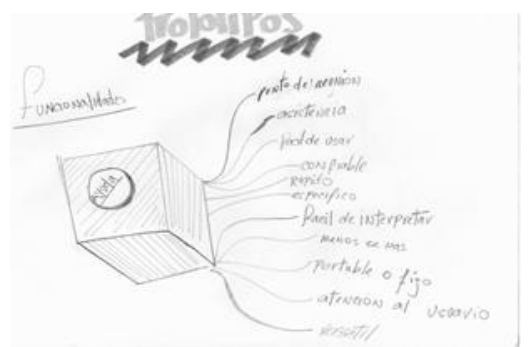

Figura 3. Primer Sketch en Papel Aplicando el Feedback de Entrevistas Anteriores

Posteriormente se determinaron 3 factores de forma que consideramos de acuerdo a las respuestas que recibimos de los sketches: una caja inalámbrica que se pueda llevar en el bolsillo o bolsa, un brazalete UNBO y un panel estático que se pueda instalar en variedad de superficies. Se crearon prototipos de baja fidelidad en cartulina y nuevamente los llevamos a nuestros grupos de enfoque para ser evaluados.
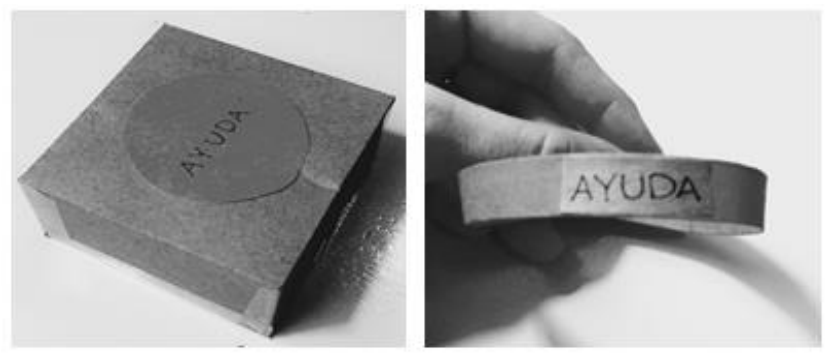

Figura 4. Primera Representación de Prototipos Físicos

Luego de determinar dimensiones adecuadas, se llevaron los prototipos tentativos a ser modelados en 3D en SketchUP [5] donde se agregó SOS en braille a los modelos portátiles ya que un integrante de nuestro grupo de enfoque, siendo ciego y sin contexto adicional no sabía el funcionamiento de UNBO y proporcionando este texto en braille se soluciona el problema de inclusión de personas ciegas en áreas sociales que no están preparadas para atender a visitantes con discapacidad visual.

Llegando a representaciones digitales de alta fidelidad, se hicieron últimas pruebas de ergonomía con modelos impresos en 3D que representaban fielmente las dimensiones de los distintos modelos y simulaban los materiales y colores que va a tener la versión final de UNBO, un botón rojo portátil o estático que llama la atención del usuario y puede ser discreto al mismo tiempo que el rojo denota urgencia en caso de una emergencia real.
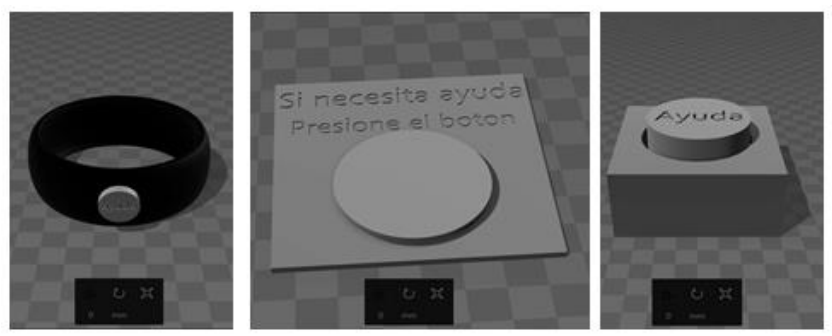

Figura 5. Modelos en 3D de Prototipos

\subsection{Comprende la experiencia entera}

Cabe resaltar que gran parte de la experiencia del usuario recae en el componente humano, por lo cual más adelante será diseñado un programa de capacitación a colaboradores de los distintos entornos donde UNBO puede ser utilizado. En fases de entrevista se hizo evidente que gran parte de adultos mayores creen que se necesita mayor cuidado y respeto hacia sus personas en entonos de comercio por lo cual parte del diseño incluye esta faceta.

\subsection{Evaluación del usuario}

Durante la duración del proceso de diseño iterativo, fue central en gran manera la colaboración de los adultos mayores que además de ser entrevistados y encuestados conformaron nuestros grupos de enfoque, guiándonos con experiencia a través de un área verdaderamente desconocida y probando nuestros varios prototipos y criticándolos con absoluta honestidad.

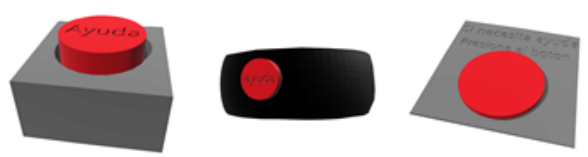

Figura 6. Modelos 3D Finales

\subsection{Multidisciplinario}

Luego de llegar a la fase final de prototipo, se hizo una versión funcional de UNBO en donde necesito conocimiento de circuitos y diseño de sistemas para lo cual se necesitó del apoyo del Ing. José Antonio Bagur que fue referente en esta área además de procesos de producción para ver la viabilidad de UNBO al momento de llevarlo al mercado.

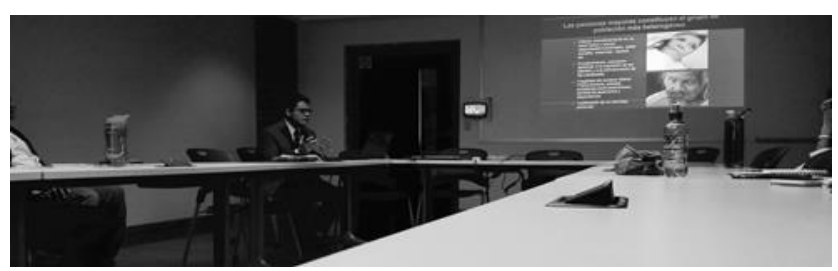

Figura 7. Charla con Dr. Javier Blanco, geriatra

Además hablamos con el Dr. Javier Blanco, psicogeriatra que fue guía esencial en ayudarnos a entender las necesidades fisiológicas, psicológicas y los cambios físicos que se debe tomar en cuenta a la hora de desarrollar productos especializados para el cuidado y protección del adulto mayor.

\section{Prototipo final}

UNBO es una plataforma de asistencia al adulto mayor en gran cantidad de factores de forma que recibe un toque para realizar el pedido de asistencia, un pulso sostenido para estado de emergencia y da respuesta al usuario a través de una LED de múltiples colores que muestra la cercanía de la ayuda pedida. Se comunica con la estación base a través de radiofrecuencia, un sistema de alcance medio y baja interferencia que permite transmitir la señal con tiempos de respuesta rápidos y de bajo costo.

\section{Factibilidad}

Parte del interés de llegar más allá de un producto puramente conceptual era ver que tan realizable era la implementación de UNBO en verdaderos ambientes donde se pensó utilizar y una consideración principal es el costo. Se cotizo el precio de fabricar más de 5000 botones funcionales, llevando la parte electrónica a una placa que utiliza bajo voltaje y una LED tri-color para dar respuesta al usuario que la ayuda viene en camino además del estado de la batería de la unidad UNBO en uso. 

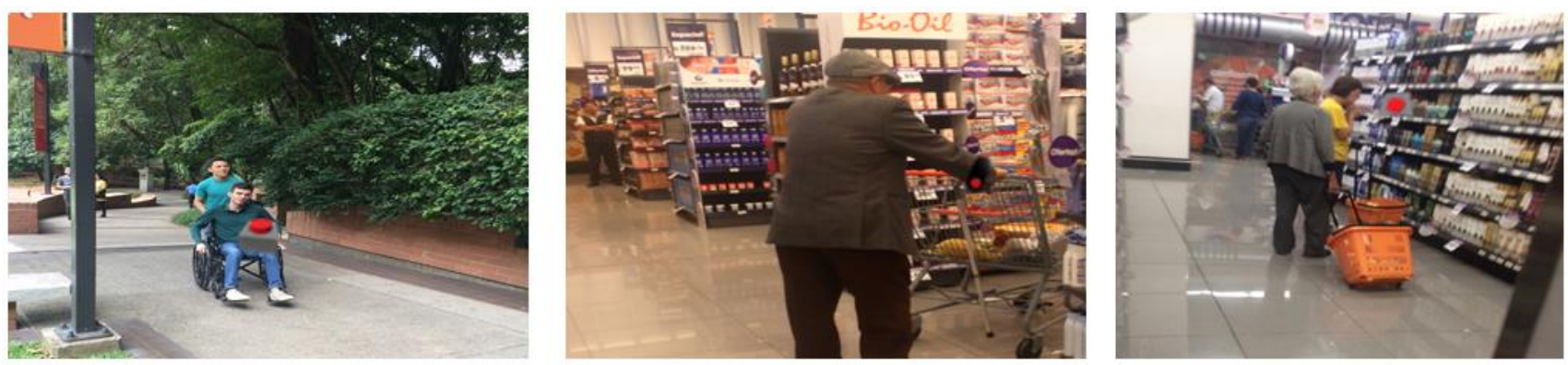

Figura 8. Composiciones Digitales de Casos de Uso de UNBO:Ayuda de Transporte en Vía Pública (izquierda), Asistencia en Comercios (centro), Emergencia Médica (derecho)

Nuestra cotización nos dice que el costo total llega a ser $\$ 5$ por unidad, el costo se distribuye en $\$ 3$ por la placa funcional y batería; el resto en la cubierta plástica de UNBO. Al saber que el sistema tendría bajo costo hablamos con gerentes de supermercados quienes mostraron interés en llevar UNBO a sus lugares de negocios.

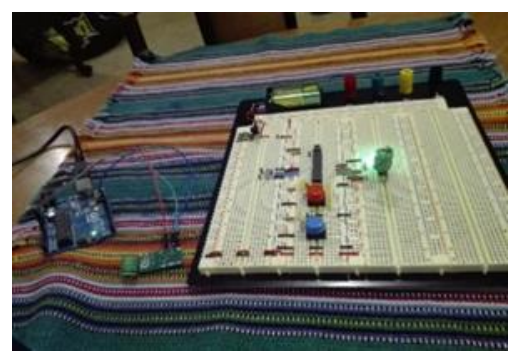

Figura 9. Placa Electrónica UNBO

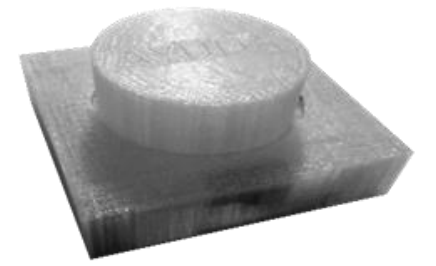

Figura 10. UNBO: prototipo funcional

\section{Conclusiones}

La vida de una persona de la tercera edad está llena de retos complejos a afrontar en el día a día, y estos son exacerbados por retos adicionales presentados por la falta de facilidades y ayuda a estos grupos.

Creamos una solución, UNBO, consistente en un sistema de unidades independientes, portátiles y de diseño atractivo que permiten al adulto mayor o persona discapacitada pedir asistencia y fácilmente recibirla por parte de personas capacitadas en esta área en especial. En nuestro proceso de diseño y sobre todo evaluación tomamos en cuenta todo tipo de personas con distintas discapacidades, integrando soluciones puntuales como el Braille en el diseño además llevando la simplicidad como axioma, guiados por las necesidades expresadas por las personas entrevistadas.

Creemos fervientemente en una solución sencilla y puntual puede crear un gran impacto positivo en la vida cotidiana de los adultos mayores en cualquier contexto de vida, en cualquier país del mundo ya que el control sobre sus necesidades está literalmente en sus manos y la ayuda esta solamente al toque de un solo botón: UNBO.

\section{Referencias}

[1] Instituto Nacional de Estadística de Guatemala: Información Demográfica de Población de Guatemala, Censo 2010. Accesado 8 - 6 - 2016, de la página del INE: 2010 http://web.archive.org/web/http://www.ine.gob.gt/index.php/ demografia-y-poblacion/42-demografiaypoblacion/207infodemo2010.

[2] Instituto Nacional de Estadística de Guatemala: Hallazgos de la Primera Encuesta Nacional de Discapacidad, 2005.

Accesado 14 - 6 - 2016, de: https://www.ine.gob.gt/sistema/uploads/2014/01/16/U318Me piRtatBurbPSoTVxo56SY9pqs0.pdf .

[3] International Organisation for Standarization: Ergonomics of Human-System Interaction - Part 11: Usability, 2015.

Accesado 14 - 6 - 2016 de http://www.iso.org/iso/catalogue_detail.htm?csnumber=6350 0

[4] T. Miaskewiecz. Personas and User Centered Design: How can Personas benefit product design processes?, 2011. Accesado 19 - 6 - 16 de Universidad de Lisboa: http://www.uselab.tuberlin.de/wiki/images/6/6d/Miaskiewicz_(2011).pdf

[5] SketchUP: The Easiest Way to Draw in 3D, 2015. Accesado 17 - 6 - 16 de página oficial http://www.sketchup.com/

[6] United Nations: World Population Ageing, 2013. Accesado Junio 16, 2015 del Department of Economic and Social Affairs - Population Division: http://www.un.org/en/development/desa/population/publicati ons/pdf/ageing/WorldPopulationAgeing2013.pdf 